hep-ph/0612184

December 2006

\title{
Two-loop virtual top-quark effect on Higgs-boson decay to bottom quarks
}

\author{
Mathias Butenschön, Frank Fugel, Bernd A. Kniehl \\ II. Institut für Theoretische Physik, Universität Hamburg, \\ Luruper Chaussee 149, 22761 Hamburg, Germany
}

\begin{abstract}
In most of the mass range encompassed by the limits from the direct search and the electroweak precision tests, the Higgs boson of the standard model preferably decays to bottom quarks. We present, in analytic form, the dominant two-loop electroweak correction, of $\mathcal{O}\left(G_{F}^{2} m_{t}^{4}\right)$, to the partial width of this decay. It amplifies the familiar enhancement due to the $\mathcal{O}\left(G_{F} m_{t}^{2}\right)$ one-loop correction by about $+16 \%$ and thus more than compensates the screening by about $-8 \%$ through stronginteraction effects of order $\mathcal{O}\left(\alpha_{s} G_{F} m_{t}^{2}\right)$.
\end{abstract}

PACS numbers: 11.10.Gh, 12.15.Ji, 12.15.Lk, 14.80.Bn 
The standard model (SM) of elementary-particle physics, whose fermion and gauge sectors have been impressively confirmed by an enormous wealth of experimental data, predicts the existence of a last undiscovered fundamental particle, the Higgs boson $H$, whose mass $M_{H}$ is a free parameter of the theory. The direct search for the Higgs boson at the CERN Large Electron-Positron Collider LEP 2 led to a lower bound of $M_{H}>114 \mathrm{GeV}$ at 95\% confidence level (CL) [1. On the other hand, high-precision measurements, especially at LEP and the SLAC Linear Collider SLC, were sensitive to the Higgs-boson mass via electroweak radiative corrections, yielding to the value $M_{H}=\left(85_{-28}^{+39}\right) \mathrm{GeV}$ together with an upper limit of $M_{H}<166 \mathrm{GeV}$ at $95 \% \mathrm{CL}$ [2]. The vacuum-stability and triviality bounds suggest that $130 \lesssim M_{H} \lesssim 180 \mathrm{GeV}$ if the $\mathrm{SM}$ is valid up to the grand-unification scale (for a review, see Ref. [3]). If the Higgs mechanism of spontaneous symmetry breaking, as implemented in the SM, is realized in nature, then we are now being on the eve of a groundbreaking discovery, to be made at the CERN Large Hadron Collider (LHC), which will go into operation in a just few months from now. After finding a new scalar particle, the burning question will be whether it is in fact the Higgs boson of the SM, or lives in some extended Higgs sector. Therefore, it is indispensable to know the SM predictions for the production and decay rates of the SM Higgs boson with high precision. Its decay to a bottom-quark pair, $H \rightarrow b \bar{b}$, is of paramount interest, as it is by far the dominant decay channel for $M_{H} \lesssim 140 \mathrm{GeV}$ (see, e.g., Ref. [4]). On the other hand, the inverse process, $b \bar{b} \rightarrow H$, was identified to be a crucial hadroproduction mechanism, appreciably enhancing the yield due to gluon fusion [5]. Precise knowledge of the bottom Yukawa coupling is also requisite for reliable predictions of associated hadroproduction of Higgs bosons and bottom quarks [6].

The purpose of this Letter is to fill a long-standing gap in our knowledge of the quantum corrections to the partial width $\Gamma_{b}$ of the $H \rightarrow b \bar{b}$ decay, by providing, in analytic form, the dominant two-loop electroweak correction, of $\mathcal{O}\left(G_{F}^{2} m_{t}^{4}\right)$, where $G_{F}$ is Fermi's constant and $m_{t}$ is the top-quark mass. This correction also applies to the cross section of $b \bar{b} \rightarrow H$. Surprisingly, it turns out to be more than twice as large as the $\mathcal{O}\left(\alpha_{s} G_{F} m_{t}^{2}\right)$ one, which is formally enhanced by one power of the strong-coupling constant $\alpha_{s}$. In the discussion of virtual top-quark effects, it is useful to distinguish between universal corrections, which are independent of the produced fermion flavor, and non-universal corrections, which are specific for the $H \rightarrow b \bar{b}$ decay because bottom is the weak-isospin partner of top. Here, we have to consider both types.

Prior to going into details with our calculation, we briefly review the current status of the radiative corrections to $\Gamma_{b}$ in the intermediate mass range, defined by $M_{W}<M_{H}<$ $2 M_{W}$. As for effects arising solely from quantum chromodynamics (QCD), the full $m_{b}$ dependence is known in $\mathcal{O}\left(\alpha_{s}\right)$ [7]. In $\mathcal{O}\left(\alpha_{s}^{2}\right)$, the leading [8] and next-to-leading [9] terms of the expansion in $m_{b}^{2} / M_{H}^{2}$ of the Feynman diagrams without top quarks are available. Those involving top quarks either contain gluon self-energy insertions or represent cuts through three-loop double-triangle diagrams; the former contribution is exactly known [10, while the four leading terms of the expansion in $M_{H}^{2} / m_{t}^{2}$ are known in the latter case [11]. In $\mathcal{O}\left(\alpha_{s}^{3}\right)$, the diagrams containing only light degrees of freedom were evaluated directly [12], while those involving the top quark were treated in the framework of an 
appropriate effective field theory [13]. As for purely electroweak corrections, the oneloop result is completely known [14. At two loops, the dominant universal correction, of $\mathcal{O}\left(G_{F}^{2} m_{t}^{4}\right)$, was already studied in Ref. [15], while the non-universal one is considered here for the first time. As for mixed corrections, the universal [16] and non-universal [17] $\mathcal{O}\left(\alpha_{s} G_{F} m_{t}^{2}\right)$ terms at two loops and the universal [18] and non-universal [19] $\mathcal{O}\left(\alpha_{s}^{2} G_{F} m_{t}^{2}\right)$ terms at three loops are available.

We now outline the course of our calculation and exhibit the structure of our results. Full details will be presented in a forthcoming communication [20]. For convenience, we work in 't Hooft-Feynman gauge. As usual, we extract the ultraviolet divergences by means of dimensional regularization, with $D=4-2 \epsilon$ space-time dimensions and 't Hooft mass scale $\mu$. We do not encounter ambiguities related to the treatment of $\gamma_{5}$ in $D$ dimensions and are thus entitled to use the anti-commuting definition. We adopt Sirlin's formulation of the electroweak on-shell renormalization scheme [21], which uses $G_{F}$ and the physical particle masses as basic parameters. We take the Cabibbo-KobayashiMaskawa quark mixing matrix to be unity, which is well justified because the third quark generation is, to good approximation, decoupled from the first two [22]. For convenience, we renormalize the Higgs sector by introducing counterterm vertices involving tadpole and Higgs-boson mass counterterms, $\delta t$ and $\delta M_{H}$, respectively [23]. Specifically, $\delta t$ is adjusted so that it exactly cancels the sum of all one-particle-irreducible tadpole diagrams.

Detailed inspection reveals that, to the orders considered here, the amputated matrix element of $H \rightarrow b \bar{b}$ exhibits the simple structure

$$
\mathcal{A}=A+B(\not p-\not p) \omega_{-},
$$

where $\omega_{ \pm}=\left(1 \pm \gamma_{5}\right) / 2$ are the helicity projection operators, $p$ and $\bar{p}$ are the four-momenta of $b$ and $\bar{b}$, respectively, and $A$ and $B$ are Lorentz scalars. Including the wave-function renormalizations of the external particles and employing the Dirac equation, we find the transition matrix element to be

$$
\mathcal{T}=\sqrt{Z_{H}}\left(\sqrt{Z_{b, L} Z_{b, R}} A+m_{b} Z_{b, L} B\right) s,
$$

where $s=\bar{u}(p, r) v(\bar{p}, \bar{r})$, with $r$ and $\bar{r}$ being spin labels. Owing to parity violation, the left- and right-handed components of the bottom-quark field, $b_{L, R}=\omega_{\mp} b$, participate differently in the electroweak interactions and thus receive different wave-function renormalizations, $Z_{b, L / R}$. At tree-level, we have $A^{(0)}=-m_{b} / v$ and $B^{(0)}=0$, where $v=2^{-1 / 4} G_{F}^{-1 / 2}$ is the Higgs vacuum expectation value. Here and in the following, superscripts enclosed in parentheses denote the loop order. In Sirlin's formulation of the electroweak on-shell scheme, where Fermi's constant is introduced to the SM through a charged-current process, namely muon decay, the $\mathrm{SU}(2)$ gauge coupling $g=2 M_{W} / v$ does not receive power corrections in $m_{t}$, so that 24 ]

$$
\frac{M_{W, 0}}{v_{0}}=\frac{M_{W}}{v}
$$

to the orders considered here, which implies that the renormalization of $v$ is reduced to the one of $M_{W}$. Here and in the following, bare quantities carry the subscript 0 . It hence 
follows that we need to perform a genuine two-loop renormalization of $Z_{H}, m_{b}, Z_{b, L / R}$, and $M_{W}$, while a one-loop renormalization of $M_{H}$ and $m_{t}$ is sufficient. As usual, we denote the sums of all one-particle-irreducible $H, f(f=b, t)$, and $W$ self-energy diagrams at four-momentum transfer $q$ as $i \Sigma_{H}\left(q^{2}\right), i\left[q\left(\omega_{-} \Sigma_{f, L}\left(q^{2}\right)+\omega_{+} \Sigma_{f, R}\left(q^{2}\right)\right)+m_{f, 0} \Sigma_{f, S}\left(q^{2}\right)\right]$, and $-i\left[\left(g^{\mu \nu}-q^{\mu} q^{\nu} / q^{2}\right) \Sigma_{W, T}\left(q^{2}\right)+\left(q^{\mu} q^{\nu} / q^{2}\right) \Sigma_{W, L}\left(q^{2}\right)\right]$, and split the bare masses as $M_{H / W, 0}^{2}=$ $M_{H / W}^{2}+\delta M_{H / W}^{2}$ and $m_{f, 0}=m_{f}+\delta m_{f}$. Imposing the on-shell renormalization conditions on the dressed propagators then yields

$$
\begin{aligned}
\delta M_{H}^{2} & =\Sigma_{H}\left(M_{H}^{2}\right), \\
Z_{H} & =\frac{1}{1+\Sigma_{H}^{\prime}\left(M_{H}^{2}\right)}, \\
\frac{\delta m_{f}}{m_{f}} & =\frac{1}{\sqrt{f\left(m_{f}^{2}\right)}-1,} \\
Z_{f, L / R} & =\frac{1}{\left(1+\Sigma_{f, L / R}\left(m_{f}^{2}\right)\right)\left(1-m_{f}^{2} \frac{f^{\prime}\left(m_{f}^{2}\right)}{f\left(m_{f}^{2}\right)}\right)}, \\
\delta M_{W}^{2} & =\Sigma_{W, T}\left(M_{W}^{2}\right),
\end{aligned}
$$

where

$$
f\left(q^{2}\right)=\frac{\left(1-\Sigma_{f, S}\left(q^{2}\right)\right)^{2}}{\left(1+\Sigma_{f, L}\left(q^{2}\right)\right)\left(1+\Sigma_{f, R}\left(q^{2}\right)\right)} .
$$

Relations that, to the order of our analysis, are equivalent to Eq. (4) were found in Ref. [25] using an alternative approach.

Performing a loop expansion and eliminating all bare masses, we thus obtain

$$
\begin{aligned}
& \frac{\mathcal{T}^{(0)}}{s}=A^{(0)}, \\
& \frac{\mathcal{T}^{(1)}}{s}=A^{(1)}+m_{b} B^{(1)}+A^{(0)}\left(\delta_{u}^{(1)}+X^{(1)}\right) \\
& \frac{\mathcal{T}^{(2)}}{s}=A^{(2)}+m_{b} B^{(2)}+A^{(1)} X^{(1)}+m_{b} B^{(1)} \delta Z_{b, L}^{(1)} \\
& +\left(A^{(1)}+m_{b} B^{(1)}+A^{(0)} X^{(1)}\right)\left[\delta_{u}^{(1)}+2(1-\epsilon) \frac{\delta m_{t}^{(1)}}{m_{t}}\right. \\
& \left.-\frac{\delta M_{W}^{2(1)}}{M_{W}^{2}}\right]+A^{(0)}\left[\delta_{u}^{(2)}+X^{(2)}+\frac{1}{2} \frac{\delta m_{b}^{(1)}}{m_{b}}\left(\delta Z_{b, L}^{(1)}\right.\right. \\
& \left.\left.+\delta Z_{b, R}^{(1)}\right)-\frac{1}{8}\left(\delta Z_{b, L}^{(1)}-\delta Z_{b, R}^{(1)}\right)^{2}\right]
\end{aligned}
$$

where

$$
\delta_{u}^{(1)}=\frac{1}{2} \delta Z_{H}^{(1)}-\frac{1}{2} \frac{\delta M_{W}^{2(1)}}{M_{W}^{2}}
$$




$$
\begin{aligned}
\delta_{u}^{(2)}= & \frac{1}{2} \delta Z_{H}^{(2)}-\frac{1}{2} \frac{\delta M_{W}^{2(2)}}{M_{W}^{2}}+\delta_{u}^{(1)}\left[-\frac{1}{2} \delta_{u}^{(1)}+2(1-\epsilon)\right. \\
& \left.\times \frac{\delta m_{t}^{(1)}}{m_{t}}-2 \frac{\delta M_{W}^{2(1)}}{M_{W}^{2}}\right]-\frac{1}{2}\left(\frac{\delta M_{W}^{2(1)}}{M_{W}^{2}}\right)^{2}
\end{aligned}
$$

are the universal corrections and

$$
X^{(i)}=\frac{\delta m_{b}^{(i)}}{m_{b}}+\frac{1}{2}\left(\delta Z_{b, L}^{(i)}+\delta Z_{b, R}^{(i)}\right) .
$$

The Feynman diagrams contributing to $A_{0}^{(2)}$ and $B_{0}^{(2)}$ are depicted in Fig. 1. They are generated and drawn using the program FeynArts [26] and evaluated using the program MATAD [27], which is written in the programming language FORM [28], by applying the asymptotic-expansion technique (for a careful introduction, see Ref. [29]). Here, $\chi$ and $\phi$ denote the neutral and charged Higgs-Kibble ghosts with masses $M_{Z}$ and $M_{W}$, respectively. The crosses in Figs. 1(s) and (t) indicate the insertions of the Higgs-boson mass and tadpole counterterms $i \delta t / v_{0}$ and $-i\left(\delta t / v_{0}+\delta M_{H}^{2}\right) / v_{0}$ in a $\phi$-boson line and a $H \phi \phi$ vertex, respectively. In the soft-Higgs limit, $M_{H} \ll m_{t}$, which is underlying our analysis, the diagrams in Figs. 1(a)-(s) can also be evaluated by applying a low-energy theorem (see Ref. [30] and references cited therein) to the corresponding $b$-quark selfenergy diagrams that emerge by removing the external Higgs-boson line. This provides a powerful check for our calculation. Apart from the diagrams in Fig. 1, we also need to calculate the relevant one-particle-irreducible $H, b$, and $W$ self-energy diagrams at two loops. Furthermore, we need to expand all the relevant one-loop diagrams through $\mathcal{O}(\epsilon)$.

We are now in a position to present our final results for the universal correction parameter $\delta_{u}$ and the relative correction to $\Gamma_{b}$. They read

$$
\begin{aligned}
& \delta_{u}=x_{t} N_{c} \frac{7}{6}+x_{t}^{2} N_{c}\left(\frac{29}{2}-6 \zeta(2)+N_{c} \frac{49}{24}\right) \\
& \quad+x_{t} \frac{\alpha_{s}}{\pi} C_{F} N_{c}\left(\frac{19}{12}-\frac{\zeta(2)}{2}\right), \\
& \frac{\Gamma_{b}}{\Gamma_{b}^{(0)}}=x_{t}\left(-6+N_{c} \frac{7}{3}\right)+x_{t}^{2}\left[-20+N_{c}(29-12 \zeta(2))\right. \\
& \left.\quad+N_{c}^{2} \frac{49}{9}\right]+x_{t} \frac{\alpha_{s}}{\pi} C_{F}\left[-36+N_{c}\left(\frac{157}{12}-\zeta(2)\right)\right],
\end{aligned}
$$

where $N_{c}=3$ and $C_{F}=\left(N_{c}^{2}-1\right) /\left(2 N_{c}\right)=4 / 3$ are color factors, $x_{t}=\left(G_{F} m_{t}^{2}\right) /\left(8 \pi^{2} \sqrt{2}\right)$, $\zeta(2)=\pi^{2} / 6$, and

$$
\Gamma_{b}^{(0)}=\frac{\sqrt{2} N_{c} G_{F} M_{H} m_{b}^{2}}{8 \pi}\left(1-\frac{4 m_{b}^{2}}{M_{H}^{2}}\right)^{3 / 2} .
$$

If we convert Eq. (9) to a mixed renormalization scheme which uses the on-shell definitions for the particle masses and the definitions of the modified minimal-subtraction $(\overline{\mathrm{MS}})$ 


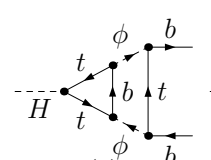

(a)

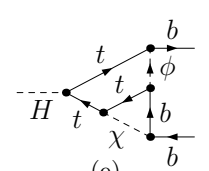

(e)

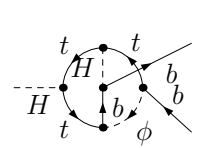

(i)
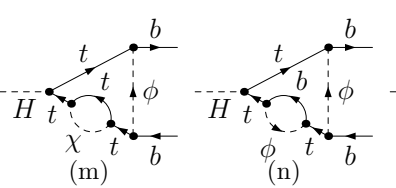

(b)

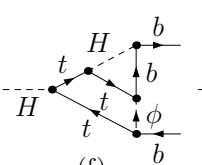

(f)

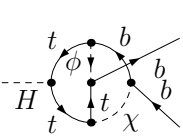

(j)

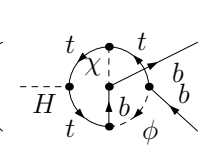

(k)

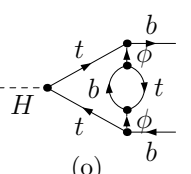

(o)

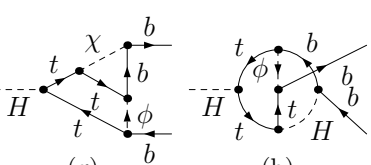

(h)

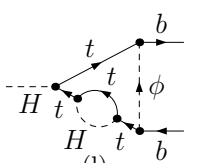

(1)
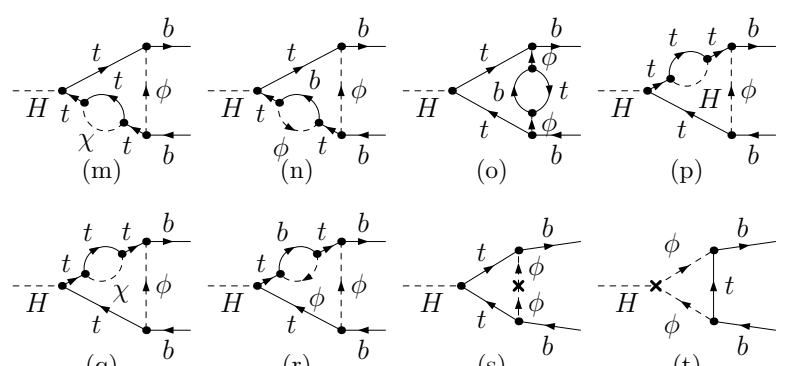

(q)

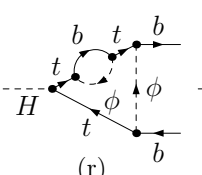

$(\mathrm{r})$

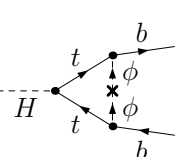

(s)

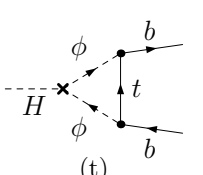

$(\mathrm{t})$

Figure 1: Diagrams contributing to $H \rightarrow b \bar{b}$ at $\mathcal{O}\left(G_{F}^{2} m_{t}^{4}\right)$.

scheme for all other basic parameters, then we find agreement with Eq. (15) for $x=0$ in the paper by Djouadi et al. [15. However, the corresponding result for the electroweak on-shell scheme presented in their Eq. (27) for $x=0$ disagrees with our Eq. (9). We can trace this discrepancy to the absence in their Eq. (25) of the additional finite term $\hat{\delta}_{u}^{(1)} \Delta \rho^{(1)}$ which arises from the renormalization of the one-loop result in their Eq. (7) according to the prescription in their Eq. (18). The $\mathcal{O}\left(G_{F}^{2} m_{t}^{4}\right)$ term in Eq. (10) represents a new result.

In Eqs. (9) and (10), we have also included the two-loop $\mathcal{O}\left(\alpha_{s} G_{F} m_{t}^{2}\right)$ corrections [16,17], which we reproduced using our calculational techniques. As for the QCD renormalization, it is understood that $m_{b}$ appearing in Eq. (11) is defined in the $\overline{\mathrm{MS}}$ scheme as $m_{b}=\bar{m}_{b}\left(M_{H}\right)$, while the electroweak part of the renormalization remains in the onshell scheme. This modification ensures that large logarithms of the type $\ln \left(M_{H}^{2} / m_{b}^{2}\right)$ that would otherwise appear already at $\mathcal{O}\left(\alpha_{s}\right)$ and spoil the convergence behavior of the perturbation expansion are properly resummed according to the renormalization group (RG) [7]. Since we wish to treat $m_{t}$ on the same footing as $m_{b}$, we adopt this mixed scheme for $m_{t}$ as well. The analysis at $\mathcal{O}\left(\alpha_{s}^{2} G_{F} m_{t}^{2}\right)$ [18,19] reveals that Eqs. (9) and (10) may be further RG-improved by taking $m_{t}$ and $\alpha_{s}$ to be $m_{t}=\bar{m}_{t}\left(m_{t}\right)$ and $\alpha_{s}=\alpha_{s}^{(6)}\left(m_{t}\right)$, respectively.

Finally, we explore the phenomenological implications of our results. Adopting from Ref. [22] the values $G_{F}=1.16637 \times 10^{-5} \mathrm{GeV}^{-2}, \alpha_{s}^{(5)}\left(M_{Z}\right)=0.1176, M_{Z}=91.1876 \mathrm{GeV}$, 
Table 1: Relative corrections to $\Gamma_{\tau}, \Gamma_{c}$, and $\Gamma_{b}$ at $\mathcal{O}\left(G_{F} m_{t}^{2}\right), \mathcal{O}\left(G_{F}^{2} m_{t}^{4}\right)$, and $\mathcal{O}\left(\alpha_{s} G_{F} m_{t}^{2}\right)$.

\begin{tabular}{|c|ccc|}
\hline Order & $\Gamma_{\tau} / \Gamma_{\tau}^{(0)}$ & $\Gamma_{c} / \Gamma_{c}^{(0)}$ & $\Gamma_{b} / \Gamma_{b}^{(0)}$ \\
\hline $\mathcal{O}\left(G_{F} m_{t}^{2}\right)$ & $+2.021 \%$ & $+2.021 \%$ & $+0.289 \%$ \\
$\mathcal{O}\left(G_{F}^{2} m_{t}^{4}\right)$ & $+0.064 \%$ & $+0.064 \%$ & $+0.047 \%$ \\
$\mathcal{O}\left(\alpha_{s} G_{F} m_{t}^{2}\right)$ & $+0.060 \%$ & $+0.452 \%$ & $-0.022 \%$ \\
\hline
\end{tabular}

and $m_{t}^{\text {pole }}=174.2 \mathrm{GeV}$ for our input parameters, so that $\alpha_{s}^{(6)}\left(m_{t}\right)=0.1076$ and $m_{t}=$ $166.2 \mathrm{GeV}$, we evaluate Eqs. (9) and (10) to $\mathcal{O}\left(G_{F} m_{t}^{2}\right), \mathcal{O}\left(G_{F}^{2} m_{t}^{4}\right)$, and $\mathcal{O}\left(\alpha_{s} G_{F} m_{t}^{2}\right)$. For comparison, we also evaluate the relative corrections to $\Gamma_{l}$ and $\Gamma_{q}$, where $l=e, \mu, \tau$ and $q=u, d, s, c$, which, to the orders considered here, are given by

$$
\begin{aligned}
& \frac{\Gamma_{l}}{\Gamma_{l}^{(0)}}=\left(1+\delta_{u}\right)^{2}-1, \\
& \frac{\Gamma_{q}}{\Gamma_{q}^{(0)}}=\left(1+\Delta_{\mathrm{QCD}}\right)\left(1+\delta_{u}\right)^{2}-1,
\end{aligned}
$$

where [7]

$$
\Delta_{\mathrm{QCD}}=\frac{\alpha_{s}}{\pi} C_{F} \frac{17}{4}
$$

is the $\mathcal{O}\left(\alpha_{s}\right)$ correction in the limit $m_{q} \ll M_{H}$.

The results are listed in Table 1. We observe that the $\mathcal{O}\left(G_{F}^{2} m_{t}^{4}\right)$ correction to $\Gamma_{b}$ increases the enhancement due to the $\mathcal{O}\left(G_{F} m_{t}^{2}\right)$ one by about $16 \%$ and has more than twice the magnitude of the negative $\mathcal{O}\left(\alpha_{s} G_{F} m_{t}^{2}\right)$ one. Also in the case of $\Gamma_{l}$, the $\mathcal{O}\left(G_{F}^{2} m_{t}^{4}\right)$ correction exceeds the $\mathcal{O}\left(\alpha_{s} G_{F} m_{t}^{2}\right)$ one. The situation is quite different for the case of $\Gamma_{q}$, which is due to the additional appearance of the sizeable product term $2 \Delta_{\mathrm{QCD}} \delta_{u}^{(1)}$ in Eq. (13).

In conclusion, we analytically calculated the dominant electroweak two-loop correction, of order $\mathcal{O}\left(G_{F}^{2} m_{t}^{4}\right)$, to the $H \rightarrow b \bar{b}$ decay width $\Gamma_{b}$ of an intermediate-mass Higgs boson, with $M_{H} \ll m_{t}$. We performed various checks for our analysis. The ultraviolet divergences cancelled through genuine two-loop renormalization. Our final result is devoid of infrared divergences related to infinitesimal scalar-boson masses. We reproduced those $H b \bar{b}$ vertex diagrams where the external Higgs boson is coupled to an internal top-quark line, which we had computed directly, through application of a low-energy theorem. After switching to a hybrid renormalization scheme, our $\mathcal{O}\left(G_{F}^{2} m_{t}^{4}\right)$ result for the universal correction $\delta_{u}$ agrees with Ref. [15]. Using our computational techniques, we also recovered the $\mathcal{O}\left(\alpha_{s} G_{F} m_{t}^{2}\right)$ corrections to $\delta_{u}$ and $\Gamma_{b}$. The $\mathcal{O}\left(G_{F}^{2} m_{t}^{4}\right)$ correction to $\Gamma_{b}$ amplifies the familiar enhancement due to the $\mathcal{O}\left(G_{F} m_{t}^{2}\right)$ correction by about $+16 \%$ and thus more than compensates the screening by about $-8 \%$ through QCD effects of $\mathcal{O}\left(\alpha_{s} G_{F} m_{t}^{2}\right)$.

We like to thank Paolo Gambino and Matthias Steinhauser for fruitful discussions. This work was supported in part by the German Federal Ministry for Education and Research BMBF through Grant No. 05 HT6GUA and by the German Research Foundation DFG through Graduate School No. GRK 602 Future Developments in Particle Physics. 


\section{References}

[1] ALEPH Collaboration, DELPHI Collaboration, L3 Collaboration, OPAL Collaboration and The LEP Working Group for Higgs Boson Searches, R. Barate et al., Phys. Lett. B 565, 61 (2003).

[2] LEP Electroweak Working Group, D. Abbaneo et al., Report No. LEPEWWG/200501; see also URL: http://lepewwg.web.cern.ch/LEPEWWG/.

[3] B.A. Kniehl, Int. J. Mod. Phys. A 17, 1457 (2002).

[4] B.A. Kniehl, Phys. Rept. 240, 211 (1994); M. Spira, Fortsch. Phys. 46, 203 (1998).

[5] F. Maltoni, Z. Sullivan, and S. Willenbrock, Phys. Rev. D 67, 093005 (2003); R.V. Harlander and W.B. Kilgore, ibid. 68, 013001 (2003); A. Belyaev, P.M. Nadolsky, and C-P. Yuan, JHEP 0604, 004 (2006).

[6] S. Dawson, C.B. Jackson, L. Reina, and D. Wackeroth, Phys. Rev. D 69, 074027 (2004); Phys. Rev. Lett. 94, 031802 (2005); Int. J. Mod. Phys. A 20, 3353 (2005); Mod. Phys. Lett. A 21, 89 (2006); E. Boos and T. Plehn, Phys. Rev. D 69, 094005 (2004); S. Dittmaier, M. Krämer, and M. Spira, ibid. 70, 074010 (2004); F. Maltoni, T. McElmurry, and S. Willenbrock, ibid. 72, 074024 (2005).

[7] E. Braaten and J.P. Leveille, Phys. Rev. D 22, 715 (1980); N. Sakai, ibid. 22, 2220 (1980); T. Inami and T. Kubota, Nucl. Phys. B179, 171 (1981); M. Drees and K. Hikasa, Phys. Lett. B 240, 455 (1990); 262, 497(E) (1991).

[8] S.G. Gorishny, A.L. Kataev, S.A. Larin, and L.R. Surguladze, Mod. Phys. Lett. A 5, 2703 (1990); Phys. Rev. D 43, 1633 (1991).

[9] L.R. Surguladze, Phys. Lett. B 341, 60 (1994).

[10] B.A. Kniehl, Phys. Lett. B 343, 299 (1995).

[11] K.G. Chetyrkin and A. Kwiatkowski, Nucl. Phys. B461, 3 (1996).

[12] K.G. Chetyrkin, Phys. Lett. B 390, 309 (1997).

[13] K.G. Chetyrkin and M. Steinhauser, Phys. Lett. B 408, 320 (1997).

[14] J. Fleischer and F. Jegerlehner, Phys. Rev. D 23, 2001 (1981); D.Yu. Bardin, B.M. Vilenskiǔ, and P.Kh. Khristova, Yad. Fiz. 53, 240 (1991) [Sov. J. Nucl. Phys. 53, 152 (1991)]; B.A. Kniehl, Nucl. Phys. B376, 3 (1992); A. Dabelstein and W. Hollik, Z. Phys. C 53, 507 (1992).

[15] A. Djouadi, P. Gambino, and B.A. Kniehl, Nucl. Phys. B523, 17 (1998). 
[16] B.A. Kniehl and A. Sirlin, Phys. Lett. B 318, 367 (1993); B.A. Kniehl, Phys. Rev. D 50, 3314 (1994); A. Djouadi and P. Gambino, Phys. Rev. D 51, 218 (1995).

[17] B.A. Kniehl and M. Spira, Nucl. Phys. B432, 39 (1994); A. Kwiatkowski and M. Steinhauser, Phys. Lett. B 338, 66 (1994); 342, 455(E) (1995).

[18] B.A. Kniehl and M. Steinhauser, Nucl. Phys. B454, 485 (1995); Phys. Lett. B 365, 297 (1996).

[19] K.G. Chetyrkin, B.A. Kniehl, and M. Steinhauser, Phys. Rev. Lett. 78, 594 (1997); Nucl. Phys. B490, 19 (1997).

[20] M. Butenschön, F. Fugel, and B.A. Kniehl (in preparation).

[21] A. Sirlin, Phys. Rev. D 22, 971 (1980).

[22] Particle Data Group, W.-M. Yao et al., J. Phys. G 33, 1 (2006).

[23] A. Denner, Fortsch. Phys. 41, 307 (1993).

[24] M. Consoli, W. Hollik, and F. Jegerlehner, Phys. Lett. B 227, 167 (1989).

[25] M. Faisst, Diploma thesis, University of Karlsruhe, 2000.

[26] T. Hahn, Comput. Phys. Commun. 140, 418 (2001).

[27] M. Steinhauser, Comput. Phys. Commun. 134, 335 (2001).

[28] J.A.M. Vermaseren, Symbolic Manipulation with FORM, (Computer Algebra Netherlands, Amsterdam, 1991).

[29] V.A. Smirnov, Applied Asymptotic Expansions in Momenta and Masses, (Springer, Heidelberg, 2001).

[30] B.A. Kniehl and M. Spira, Z. Phys. C 69, 77 (1995); W. Kilian, ibid. 69, 89 (1995). 